\title{
Diseño y validación de un cuestionario para evaluar la actitud percibida del profesor en clase y de un cuestionario para evaluar los contenidos actitudinales de los alumnos durante las clases de educación física en secundaria
}

\author{
Design and validation of a questionnaire to evaluate the perceived attitude of the professor and of a \\ questionnaire to evaluate the actitudinal contents of the students during the physical education classes in \\ secondary education
}

Enrique Ortega, Antonio Calderón, José Manuel Palao, M. C. Puigcerver

Universidad Católica de San Antonio (España)

\begin{abstract}
Resumen: El objetivo del presente estudio fue diseñar un instrumento que mida de forma válida y fiable en alumnos de enseñanza secundaria obligatoria la actitud que perciben de su profesor en clase de educación física, y otro instrumento que evalué los contenidos actitudinales de los alumnos. En primer lugar, se realizó una validación del contenido a través del acuerdo y consenso de nueve jueces expertos. En segundo lugar, se aplicó el cuestionario a una muestra de 38 sujetos, de $3^{\circ}$ de la E.S.O., con edades entre $14,6 \pm 0,8$, dos veces con un intervalo de una semana. Se calculó la fiabilidad mediante la aplicación de la prueba test-retest. Los datos iniciales aportaron modificaciones importantes realizadas tanto por los jueces expertos como por los propios sujetos. Tras realizar las modificaciones, se apreciaron adecuados de índices de validez y fiabilidad de ambos instrumentos.
\end{abstract}

Palabras clave:Actitud, validez, fiabilidad, cuestionario.

\begin{abstract}
The purpose of this paper was to design an instrument to evaluate the attitude which students of secondary education perceive their professor in physical education classes, and another instrument that evaluated the actitudinal contents of the students. Firstly, it was made a validation of the content through the agreement and consensus of nine expert judges. Secondly, the questionnaire was applied to a sample of 38 subjects, of $3^{\circ}$ of the E.S.O., with ages between $14.6 \pm 0.8$, twice with an interval of one week. The reliability by means of the application of the test calculated test-retest. The initial dates contributed important modifications made so much by the expert judges as by the own subjects. After making the modifications, validity indices of reliability of both instruments were considered adequate.
\end{abstract} Key words: Attitude, validity, reliability, questionnaire.

\section{Introducción}

La actividad fisica tiene un importante papel dentro de la formación integral de los jóvenes. La actividad física es entendida, en términos de educación activa, como «la implicación de los alumnos en almenos dos horas de práctica fisica en el marco de los colegios» (Ahrabi-Fard, y Mavienko, 2005), y la calidad de vida es entendida como el «bienestar fisico, que a su vez depende de un apropiado crecimiento, de un normal funcionamiento fisico, de unos niveles óptimos de cualidades fisicas, y del disfrute del tiempo libre de forma fisicamente activa a lo largo de la vida» (US Department of Health and Human Services, 2000). Este concepto de actividad física coincide con la contemplada por el Real Decreto 112/2000 (B.O.E., 2000), el cual señala como uno de los objetivos de las clases de educación física el fomento de la actividad física.

El periodo de edad de la adolescencia es dónde más abandono de práctica de actividad física(Sallis, Mckenzie, y Alcaráz, 1993; Weinberg, y Gould, 1995; Casimiro, 1999). Los motivos a los que se atribuyen este abandono son principalmente: la poca satisfacción personal del alumnado (Bunner, 1997; 1999; Hassandra, Gordas, y Chroni, 2003; Ntounamis, Pensgard, Martín, y Pipe, 2004; Xiang, McBride, y Guan, 2004) la escasa competencia para el desarrollo de práctica (Hassandra, y cols., 2003; Ntounamis, y cols., 2004), el escaso volumen de práctica (número de horas) respecto a actividades físicas (Graham, Soares, y Harrington, 1983; Pierón, 1988; Ericcson, 1996), la baja intensidad a la que se desarrolla la actividad física (Calvert, Ross, y Hamlin, 2001; Swain, y Franklin, 2002), y la influencia del profesor en el proceso como la figura importante en relación con la motivación del alumnado (Silverman, 1985; Castejón, Giménez, Jiménez, y López, 2003). Para reducir esa tendencia numerosos autores plantean el uso de las clases de

Fecha recepción: 12-02-08 - Fecha envío revisores: 12-02-08 - Fecha de aceptación: 25-03-08 Correspondencia: Antonio Calderón Luquin

$\mathrm{Av} /$ de los Jerónimos s/n

30107 Guadalupe Murcia (España)

E-mail: eortega $@$ pdi.ucam.edu educación física y la práctica del deporte escolar, principalmente, como medio de promoción de la actividad física saludable y de calidad (Cavill, Biddle, y Sallis, 2001; Ahrabi-Fard, y Mavienko, 2005).

Para alcanzar estos objetivos en la asignatura de educación física se debe considerar la inclusión los contenidos conceptuales, actitudinales, y procedimentales. Tradicionalmente, en las clases de educación física solamente sean controlados y evaluados los contenidos procedimentales. En menor medida se han controlado los contenidos conceptuales, y se han dejando a la experiencia del profesor el control de aspectos actitudinales.

Los distintos instrumentos más utilizados por el docente para el control del proceso de enseñanza se encuentran: los test, las pruebas, las escalas de observación, las listas de control, las hojas de registro, etc. Estos medios que emplea el docente no hacen participes a los alumnos. Sin embargo, este tipo de instrumentos descuida la percepción de los protagonistas, cuando lo que se pretende conseguir como objetivo final es ayudarles a que aumenten su competencia y autonomía. El diario del alumno es una herramienta muy empleada en el aula al aumentar la participación del alumno en su formación, pero de difícil registro desde el punto de vista científico, y de los recursos temporales que requiere (Hernández y col, 2004).

En el ámbito de la educación física, Thomas y Nelson, (2007) y Silverman y Subramanian (1999) entre otros indican, que la herramienta por excelencia es el cuestionario al tratarse de un instrumento de fácil aplicación que conlleva a su vez una pérdida de poco tiempo de aplicación. Sin embargo, en muchas ocasiones el cuestionario no registra realmente aquello que pretende medir, debido a una escasa rigurosidad científica, tanto en su diseño, como sobre todo en su validación. A pesar de ser muy utilizados, son muy pocos los cuestionarios que se diseñan $\mathrm{y}$ validan siguiendo los procesos metodológicos adecuados (Burgos, 2006; Wiersma, 2001). El propósito del presente estudio es diseñar instrumentos que midan de forma valida y fiable en alumnos de educación física de enseñanza secundaria obligatoria: a) la actitud que los alumnos perciben que tienen su profesor en clase; $y \mathrm{~b}$ ) los contenidos actitudinales de los alumnos. 


\section{Método}

\subsection{Participantes}

Para el estudio de la validez de contenido se emplearon nueve jueces expertos. Todos ellos eran profesores titulares de distintas universidades españolas, poseían el grado de doctor en ciencias de la actividad fisica y el deporte, y eran expertos en la enseñanza de los deportes. Para el estudio de la validez de comprensión y la fiabilidad, se empleo una muestra 38 alumnos, de $3^{\circ}$ de la E.S.O., del colegio privado-concertado «El taller», de Molina de Segura (Murcia), con edades entre 14,6 $\pm 0,8$. La muestra fue escogida de forma intencional (accesibilidad).

\subsection{Diseño}

Para el estudio de la validez de los cuestionarios se definió la « «validez de contenido» como el grado en que una prueba representa de forma adecuada lo que se ha realizado (Thomas y Nelson, 2007; Wiersma, 2001). Para alcanzar niveles óptimos de validez de contenido en los cuestionarios objeto de estudio, se utilizó la técnica de jueces expertos, y un estudio piloto para conocer la validez de contenido desde la perspectiva de la validez de comprensión de los sujetos objeto de estudio. Las variables objeto de estudio para valorar la validez fueron:

a) Validez de contenido mediante jueces expertos. Se solicitó a los jueces expertos que valorasen diferentes aspectos sobre la información inicial, la escala de medida, y los ítems de los diferentes cuestionarios, y una valoración global de cada uno de ellos (Wiersma, 2001). Con respecto a la información inicial, a la escala de medida, y a la valoración final se solicitó a los jueces expertos que hiciesen una valoración conceptual (grado de comprensión, adecuación en la redacción, etc.) y una valoración cuantitativa (escala de 1 a 10) de los mismos.

En relación a los ítems del cuestionario, se registró:

1. Grado de pertenencia al objeto de estudio. Se registró en qué medida cada uno de los ítems de los diferentes cuestionarios debían formar parte del mismo. Para ello, se solicitó a los jueces expertos que valorasen conceptualmente la importancia de cada uno de los ítems de los diferentes cuestionarios. En este sentido, los jueces expertos indicaban la necesidad de que el ítem formase, ono, parte del cuestionario. De igual modo, en una escala de 1 a 10 indicaban el grado de pertenencia del ítem al cuestionario. Siguiendo la propuesta de Bulger y Housner, (2007) se decidió eliminar todos aquellos ítems con valores inferiores a 7 , modificar los ítems con valores entre 7,1 y 8, y aceptar los superiores a 8,1 .

2. Grado de precisión y corrección. Se registró el grado de precisión en la definición y redacción de cada uno de los ítems.

b) Validez de comprensión. Se valoró el grado de entendimiento de los alumnos sobre cada uno de los instrumentos. Se realizó un estudio piloto en el que, tras la administración del cuestionario a los sujetos objeto de estudio, se analizaron los siguientes aspectos:

1. Grado de comprensión cualitativo del cuestionario. Para ello, se registraron las preguntas, dudas, sugerencias, etc., que los sujetos realizaron en la sesión de cumplimentación del cuestionario.

2. Grado de comprensión cuantitativo del cuestionario. Para ello, se analizaron los valores de las preguntas de auto-informe mediante una escala de 1 a 10. De igual forma, se le solicitó que indicasen aquellos ítems que no entendían tras una primera lectura.

3. Análisis de las respuestas. Para conocer el grado de comprensión de cada uno de los ítems, se analizaron las respuestas de los sujetos desde dos puntos de vista:

- Análisis de la frecuencia de la respuesta «no sabe/ no contesta». Se decidió eliminar todos aquellos ítems en los que el porcentaje de respuesta «no sabe no / contesta» fuese superior al 5\%.

- Frecuencia de respuesta elevada. Con el objetivo de que losítems de los cuestionarios discriminasen a los sujetos objeto de estudio se decidió eliminar todos aquellos ítems que presentasen la misma respuesta en más del $90 \%$ de la muestra.

c) Fiabilidad. Se definió fiabilidad (Thomas y Nelson, 2007) como reproducibilidad de una medida. Para alcanzar niveles óptimos de fiabi- lidad en los cuestionarios objeto de estudio, se utilizó la técnica de testretest (Baumgartner, 2000).

\subsection{Instrumentos}

Los instrumentos empleados fueron los cuestionarios a validar y dar fiabilidad:

a) Cuestionario de Actitud del profesor en clase (versión original y versión definitiva, anexo 1 y 2 respectivamente). El cuestionario se planteó tras revisar el instrumento creado por Ureña, Soriano, Martínez y Hernández (1997), por Papaioannou, Tsigilis, Kosmidou, y Milosis (2007), y el empleado por Calderón, Palao, y Ortega (2005). El cuestionario poseía una breve introducción y un bloque de preguntas de carácter sociodemográfico. Se registraban tres grupos de variables: el primero en relación a la percepción del dominio sobre la materia que mostraba el profesor, con cinco preguntas cerradas (con una escala de no adecuado, regular, bueno, y muy bueno, de 0 a 4). El segundo sobre la precepción de la evaluación que realizaba, con dos preguntas (con una escala de no adecuado, regular, bueno y muy bueno, de 0 a 4). El tercero, sobre cómo se percibe al profesor en las relaciones sociales dentro de clase, con cinco preguntas de las cuales dos fueron cerradas (con una escala de no adecuado, regular, bueno, y muy bueno, de 0 a 4 ) y tres abiertas.

b) Escala para evaluar la actitud del alumno en clase (versión original y versión definitiva, anexo 3 y 4 respectivamente). El cuestionario fue de elaboración propia. Se realizó a partir de los contenidos mínimos de enseñanza a llevar a cabo en la ESO establecidos por el Decreto $112 \mathrm{de}$ 12 de septiembre de 2002 del Boletín Oficial de la Región de Murcia (B.O.R.M., 2002) y por el Real Decreto 1631/2006 de 29 de diciembre (B.O.E., 2006). El cuestionario constaba de una breve introducción; un bloque de preguntas de carácter sociodemográfico con preguntas cerradas y abiertas, sobre el curso escolar al que pertenecían, el género y la fecha de nacimiento; y seis bloqueos de preguntas sobre la actitud. Las variables que se aborda el cuestionario atendían las normas y reglamento, el calentamiento previo, las posibilidades físicas y capacidades de superación personal, la ayuda y cooperación entre compañeros y profesor, el efecto de la práctica sobre la salud, y la actitud ante la clase de educación física. Las variables se registraban mediante diferentes preguntas cerradas cada uno, excepto en el bloque sobre actitud ante la clase de educación física en donde se realizan preguntas cerradas y una abierta. Con el objetivo de evitar el sesgo de aquiescencia no todas las preguntas tienen la misma dirección, por lo que la escala estará invertida (ítems $5,6,815,18,20,21$, y 22$)$

\subsection{Procedimiento}

Los cuestionarios se construyeron en cinco fases, siguiendo las indicaciones de Carretero-Dios y Pérez (2007). La primera fase, consistía en el diseño de cada uno de los cuestionarios, teniendo en cuenta los objetivos marcados para cada instrumento (anexos 1 y 3 ). Inicialmente se desarrolló un planteamiento, acorde a Heinemann (2003), y Herrero (2002), que consistía en desarrollar una secuencia apropiada a la planificación de un instrumento de medición. Todos los instrumentos constaban de una breve introducción inicial, adaptada de Magalhães y Hill (2005), en la cual se registraban los datos (anónimos), de identificación y de clasificación de la muestra. Además de mencionar los objetivos, y las instrucciones para contestar de forma correcta. A continuación, la escala empleada con su explicación (si era necesario) y los ítems concretos. Para los cuales se realizó una breve revisión bibliográfica, como indica Thomas, y Nelson (2007), de cuestionarios similares, temáticas acordes y/o con características comunes. Todo ello, con el objetivo de dotar a los instrumentos de coherencia interna, produciéndose adaptaciones de los ítems según la estructura de cada cuestionario. Al finalizar los ítems, se incluyeron dos preguntas con carácter de auto-informe: 1) sobre la dificultad y/o facilidad en responder; y 2) sobre los ítems que le hayan resultado poco entendibles.

La segunda fase, tenía por objetivo adquirir la validez de contenido. Para ello, se enviaron los cuestionarios a un grupo de jueces expertos. Cada juez experto respondió el cuestionario en función a los criterios 


\begin{tabular}{|c|c|c|c|c|c|c|c|c|c|c|c|c|c|}
\hline & \multirow{2}{*}{$\begin{array}{c}\text { Información } \\
\text { previa }\end{array}$} & \multicolumn{11}{|c|}{ ÍTEMS } & \multirow{2}{*}{$\begin{array}{c}\text { Valoración } \\
\text { global }\end{array}$} \\
\hline & & 1 & 2 & 3 & 4 & 5 & 6 & 7 & 8 & 9 & 10 & 11 & \\
\hline Expertos & 9 & 9 & 6,5 & 7,3 & 9,2 & 8,8 & 7 & 7,1 & 6,2 & 8,5 & 5,2 & 4,5 & 7,26 \\
\hline Acción & no & no & $\mathrm{e}$ & si & no & no & si & si & $\mathrm{e}$ & no & $\mathrm{e}$ & $\mathrm{e}$ & \\
\hline
\end{tabular}

Leyenda: sobre la acción que se realizó, si el valor obtenido era superior a 8 (no), si el valor obtenido era de 7 o superior, el ítem fue revisado y modificado (si); si el valor era inferior a 7 , se suprimía o cambiaba por otro (e).

marcados. La tercera fase, supuso la interpretación de las respuestas de cada uno de los nueve jueces expertos, tras las cuales se modificaron algunos aspectos de los cuestionarios.

En la cuarta fase, se realizó un estudio piloto, en el que se analizó la validez de contenido (validez de comprensión) de los alumnos, así como la fiabilidad de los cuestionarios (prueba test-retest). Se administraron los instrumentos en dos ocasiones, siguiendo las propuestas de Nevil, Lane, Kilgour, Bowes y Whyte (2001). De igual modo, siguiendo la propuesta de Baumgartner (2000), ambas mediciones estuvieron separadas en el tiempo, por una semana, en circunstancias prácticamente idénticas. En la quinta fase, se analizaron los datos. Se obtuvieron así las conclusiones necesarias para que se produjese el diseño de los cuestionarios definitivos (anexo 2 y 4 ).

\subsection{Estadística}

El análisis estadístico de los datos se realizó con el paquete informático SPSS versión 14.0. En primer lugar, se realizó un análisis descriptivo. Posteriormente, con el objetivo de conocer la fiabilidad para las variables continuas, se calculó el coeficiente de correlación intraclase, y para las variables categóricas, el índice de Kappa siguiendo los valores de referencia deAltman (1991).

\section{Resultados}

A) Cuestionario de la percepción de la actitud del profesor/a en clase

Con respecto a la validez de contenido, los jueces expertos valoraron la introducción inicial de forma positiva. Su valoración media fue de 10 puntos. Los jueces expertos no realizaron valoraciones sobre ninguna de las escalas de medida empleadas, indicando un valor medio de 9.7. En relación con los ítems del cuestionario, la valoración de los ítems fue apreciada con una adecuada formulación y un objeto de estudio acorde al objetivo marcado para el instrumento. Sin embargo, se aprecian aportaciones con respecto a términos, enunciaciones adecuadas conceptualmente, y el tipo de ítem abierto empleado. Así, el juez 4 incide añadiendo que, «no queda muy claro el término «en relación con los alumnos», "¿se refiere al trato personal?», "¿a la forma en que se relaciona con los alumnos?», "habria que diferenciar entre exposiciones teóricas y prácticas». «Tal vez no plantearía así esta pregunta. Quizá con lo que destaquen y lo que menos les gusta ya se tendrían criterios para saber lo que sería recomendable que tuviese en cuenta el profesor», etc., señaló el juez 1, El juez 3, indicó que, «las preguntas abiertas son dificiles de codificar después para el análisis de los datos».

La valoración global del cuestionario fue positiva. No obstante, varios expertos, señalan que «a pesar de la estructura seguida en base a 1) Relaciones sociales, 2) Dominio de la materia, 3) Evaluación, etc., deben mejorarse las preguntas para que no den respuestas tan generales» (jueces 3, 4, y 6). En la tabla 1 se puede observar la valoración numérica del cuestionario. La valoración global fue de 7,26. Se aprecia una valoración baja en los ítems 2 , y 8 . Además se encuentra, que los dos últimos ítems 10 y 11 obtienen una valoración muy inferior a lo exigido (5,2 y 4,5, respectivamente). En cualquier caso, los jueces expertos indicaban que la baja valoración de dichos ítems, se debía a que eran preguntas abiertas, y que las preguntas abiertas dificultaban mucho la evaluación y control de las respuestas, ya que necesitaría un análisis y recodificación de cada una de las respuestas. De igual modo indicaban que eran preguntas muy interesantes, así como que su grado de compresión era muy adecuado.

Desde la perspectiva de la validez de comprensión, con respecto al grado de compresión cualitativo, los alumnos objeto de estudio no realizaron ninguna pregunta sobre el cuestionario. Con respecto al grado de compresión cuantitativo, se observó una media de 7,34 en la valoración del auto-informe del cuestionario. Por otro lado, con respecto a la dificultad de compresión de cada uno de los ítems, resaltó el ítem 8 , con un $21,05 \%$, de sujetos indicaban que se trataba de un ítem de dificil compresión. Igualmente, remarcar que el análisis de las dos últimas preguntas se corresponde con un análisis más exhaustivo y complejo, por el repertorio de posibles respuestas.

Tras analizar las respuestas en los ítems que obtuvieron por respuesta «no sabe / no contesta», se encontró que no se produjo ningún dato de relevancia. Finalmente cabe destacar que ninguna de las categorías de los diferentes ítems del cuestionario, obtuvo un porcentaje de respuesta superior al $90 \%$. En cualquier caso, se apreciaron ítems con porcentajes elevados con respecto al resto, como los ítems 3 y 9 , con un $52,63 \%$ y un $57,89 \%$ respectivamente.

En la tabla 2, se presentan los datos obtenidos sobre fiabilidad en el cuestionario de actitud del profesor/a en clase (tabla 2). Sólo el ítem 3 presento un valor inferior a lo recomendado por Altman (1991).

b) Cuestionario-escala de la actitud en clase

Con respecto a la validez de contenido, los jueces expertos valoraron la introducción inicial de forma positiva (nota media de 9,3). Se sugiere realizar una modificación. Así el juez 3 indico que, «la introducción considero que podría ser revisada», «La actitud, es uno de los elementos que conforman el proceso de E-A. Creo que habría que concretar que pretende este cuestionario y relacionarlo adecuadamente con el título del mismo».

En relación a la escala de medida, los jueces expertos no hicieron ningún tipo de aportación en este sentido, apreciándose una media de 10 puntos. Con respecto a los ítems del cuestionario, se aprecia una adecuada formulación y un objeto de estudio preciso. Las únicas matizaciones proporcionadas fueron en cuanto a la adecuación y precisión del texto en las posibles respuestas y a correcciones gramaticales. En concreto para el ítem 2, el juez 7 comentó «creo que sería más adecuada su

\begin{tabular}{lc}
\hline \multicolumn{2}{c}{ Tabla 2. Valores obtenidos sobre la fiabilidad del cuestionario de la actitud } \\
del profesor/a en clase. \\
\hline \multicolumn{1}{c}{ Variables } & Fiabilidad \\
\hline 1.- Explicando la materia lo consideras & 0,7 \\
2.- Explicando la materia en clase práctica lo consideras & 0,71 \\
3.- Realizando una demostración de la destreza lo consideras & $0,45^{*}$ \\
5.- Con el control y la organización de la clase lo consideras & 0,72 \\
6.- El procedimiento de evaluación que desarrolla es & 0,69 \\
7.- La forma en que se relaciona con los alumnos es & 0,88 \\
8.- La atención a los problemas individuales es & 0,75 \\
9.- En las clases el clima de convivencia que crea es & 0,72 \\
10.- pregunta abierta 1 & \\
11.- pregunta abierta 2 & \\
12.- pregunta abierta 3 & \\
\hline
\end{tabular}

Leyenda: $* * * 0,2$ fuerza pobre; $* * 0,21-0,4$ fuerza débil; $* 0,41-0,6$ fuerza moderada; 0,61-0.8 fuerza buena; 0,81-1 fuerza muy buena. Valores obtenidos de Altman, (1991). 


\begin{tabular}{|cccccccccc|}
\hline \multicolumn{7}{|c|}{ Tabla 3. Promedio de la valoración de los expertos sobre el cuestionario-escala sobre las actitudes en clase. } \\
\cline { 3 - 8 } & \multirow{2}{*}{ Información previa } & \multicolumn{7}{c|}{ ÍTEMS } & \multirow{2}{*}{ Valoración global } \\
\cline { 3 - 8 } & & $\mathbf{1}$ & $\mathbf{2}$ & $\mathbf{3}$ & $\mathbf{4}$ & $\mathbf{5}$ & $\mathbf{6}$ & 7,64 \\
\hline Expertos & 4,5 & 8 & 6,75 & 9 & 8,25 & 9 & 8,3 & \\
\hline Acción & si $^{*}$ & no & $\mathrm{e}$ & no & no & no & no \\
\hline
\end{tabular}

Leyenda: sobre la acción que se realizó, si el valor obtenido era superior a 8 (no = no se intervino), si el valor obtenido era de 7 o superior, el ítem fue revisado y modificado (si); si el valor era inferior a 7, se suprimía o cambiaba por otro (e).

redacción de la siguienteforma: «No lo considero necesario, por lo que cuando practico actividad fisica pormi propia cuenta no lo realizo; sólo lo pongo en práctica cuando el profesor lo propone en las clases de educación fisica». Igualmente, para el ítem 4, «la actitud de ayuda y cooperación puede estar centrada en «ganar» o en «facilitar aprendizajes». Sería interesante tenerlo presente» indicó el juez 5.

A nivel general, los jueces expertos apuntan que la estructura del cuestionario no es excesivamente acorde entre el objetivo de estudio y el contenido. En concreto, se señaló, «en el cuestionario se incluyen preguntas que indagan sobre aspectos diversos: actitudes, valores, hábitos, pero no veo que se ciña al enunciado que habéis puesto; de hecho creo que hay poca relación entre tantos aspectos» (juez 1). El juez 8, respecto al contenido del cuestionario remarcó, «creo que se pueden hacer las preguntas de forma general hacia cualquier disciplina deportiva, y luego buscar la forma de obtener información más específica relacionada con un deporte, si es lo que se pretende, obien, hacer todas las preguntas de forma especifica orientadas a un deporte especifico».

En la tabla 3 se puede observar la valoración cuantitativa del cuestionario. Se aprecia una valoración global de 7,64. En concreto, se aprecia una valoración inferior a lo permitido en la información previa y en el ítem 2 , con 6,75 .
Con respecto al grado de compresión cualitativo, los sujetos realizaron preguntas en los ítems de los bloques 4 y 5 , que denotaban falta de compresión. Así por ejemplo indicaron: «iporqué sólo quiere una respuesta a las preguntas? yo creo que podrían ser varias y lo entiendo bien». También, en relación a los efectos de práctica habitual de actividad física "¿la información de la pregunta no la tengo muy clara?, ¿no entiendo bien la pregunta?, la contesto».

Desde la perspectiva del grado de compresión cuantitativo, se observó una media de 7,8 en la valoración del auto-informe del cuestionario. Por otro lado, cabe destacar que un $46 \%, 44 \%$ y $49 \%$ de los sujetos indicaron que en el ítem 4,5 y 6 respectivamente, existía cierto grado de dificultad en su compresión.

En el análisis sobre las preguntas «no sabe / no contesta», no se obtuvo ningún resultado que permitiera la eliminación del ítem del cuestionario. Finalmente cabe destacar que ninguna de las categorías de los diferentes ítems del cuestionario, obtuvo un porcentaje de respuesta superior al $90 \%$. En cualquier caso, se apreciaron ítems con porcentajes elevados con respecto al resto, como el ítem 1 y 2 de la pregunta 4 con un $65,79 \%$ y $57,89 \%$ respectivamente, el ítem 2 de la pregunta 5 con un $63,16 \%$, el ítem 2 de la pregunta 6 , con un $57,89 \%$.

\begin{tabular}{|c|c|}
\hline 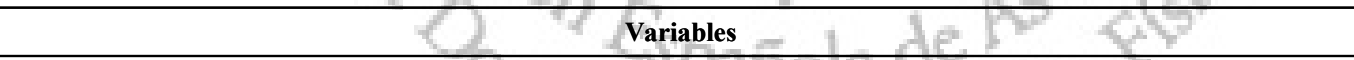 & Fiabilidad \\
\hline 1_1 Es preciso seguir el reglamento y las normas de juego. (Aunque esto no me permita ganar siempre). & 0,69 \\
\hline 1_2 Ganar en un equipo o juego de baloncesto, solo tiene mérito cuando se respetan las reglas del juego. & 0,67 \\
\hline 1_3 Los árbitros siempre tratan de emitir sus juicios sin favorecer a nadie. & 0,74 \\
\hline 2_1 Hago un buen calentamiento, porque así preparo mi cuerpo para afrontar la actividad posterior. & 0,69 \\
\hline 2_2 Normalmente, sólo hago ejercicios de calentamiento cuando hace frío. & 0,68 \\
\hline $\begin{array}{l}\text { 2_3 Sólo realizo el calentamiento en clase de educación física con el profesor, cuando hago ejercicios por mi cuenta, } \\
\text { considero que no hace falta. }\end{array}$ & 0,72 \\
\hline 3_1 Siempre me esfuerzo para tratar de aprender habilidades y gestos nuevos en baloncesto. & 0,88 \\
\hline 3_2 Me desanimo frecuentemente porque no me sale lo que el profesor plantea en clase de educación física. & 0,65 \\
\hline 3_3 Soy capaz de resolver las tareas difíciles si me esfuerzo lo suficiente y lo sé. & 0,72 \\
\hline 3_4 Gracias a mis habilidades y capacidades puedo superar situaciones inesperadas en la práctica de actividades físicas. & 0,65 \\
\hline 3_5 Mientras que realizo práctica deportiva soy capaz de controlar la situación. & 0,78 \\
\hline 4_1 Ayudo a mis compañeros siempre que necesitan ayuda mediante consejos, ánimo, correcciones, ayudas manuales, etc. & 0,77 \\
\hline $4 \_2$ Ayudo al profesor a sacar y guardar el material, ya que eso también es labor nuestra. & 0,71 \\
\hline 4_3 Pase lo que pase durante la práctica deportiva soy capaz de mantener valores de compañerismo. & 0,87 \\
\hline 5_1 Hago deporte y lo practico siempre que puedo ya que favorecerá mi salud. & 0,70 \\
\hline 5_2 La actividad física y deportiva sólo la práctico por obligación, no creo que favorezca mi salud. & $0,36^{* *}$ \\
\hline $5 \_3$ Me encanta la actividad deportiva pero solo para verla como espectador. & 0,62 \\
\hline 5_4 Me encanta la actividad deportiva pero no puedo practicarla por falta de tiempo. & 0,85 \\
\hline 6_1 Voy pensando que voy a mejorar mi estado de ánimo. & 0,70 \\
\hline $6 \_2$ Voy pensando que el profesor-a suele hacerme poco caso. & $0,62^{*}$ \\
\hline $6 \_3$ Voy pensando que es un buen momento de relacionarme con los demás. & $0,45^{* *}$ \\
\hline $6 \_4$ Voy pensando que el profesor-a suele animarme para participar en las actividades. & $0,48^{* *}$ \\
\hline $6 \_5$ Voy pensando que es importante para mi desarrollo como persona. & 0,76 \\
\hline
\end{tabular}

Leyenda: ${ }^{* * *}<0,2$ fuerza pobre; ${ }^{* *} 0,21-0,4$ fuerza débil; ${ }^{* 0,41-0,6}$ fuerza moderada; 0,61-0.8 fuerza buena; 0,81-1 fuerza muy buena. Valores obtenidos de Altman, (1991). 
Con respecto a la fiabilidad, en la tabla 4 se aprecian los valores de la fiabilidad (prueba test, re-test), obtenido para cada uno de los ítems. El ítem 2 del bloque 5 de preguntas, presenta un 0,36 un valor muy inferior a lo exigido. Igualmente, los ítems 2,3 y 4 , del bloque de preguntas 6 , muestran valores bajos que no alcanzan lo exigido $(0,62,0,45, y$ 0,48 respectivamente).

\section{Discusión}

Desde una perspectiva general, se apreciaron interesantes aportaciones por parte de los jueces expertos en ambos cuestionarios. Estas aportaciones están principalmente vinculadas a la mejora de la compresión de los ítems y escalas de medida por parte de los alumnos, aunque en algunos casos se hace alusión a la necesidad de eliminar ítems e incluso añadir algunos nuevos. Al analizar la validez con respecto al grado de comprensión de los sujetos, cabe destacar la enorme necesidad de realizar estudios pilotos en los que, una vez realizados los cambios propuestos por los jueces expertos, se administren los cuestionarios in situ. Los datos obtenidos en el presente estudio, indican que, a pesar de que los jueces expertos indiquen que el ítem es correcto, y que su redacción y grado de comprensión es elevado, puede ocurrir que los sujetos que deben contestar el cuestionario no perciban el mismo criterio (Bulger y Housner, 2007; Zhu, Ennis, y Chen, 1998). De igual modo, resulta de elevado interés analizar el grado de respuestas «no sabe/no contesta», así como analizar todas aquellas repuestas con porcentajes de aparición superiores al 90\%. El grado de respuestas «no sabe/no contesta» afianza la información obtenida en las preguntas orales que los sujetos indican, si bien, permiten eliminar el sesgo de preguntar en voz alta las dudas. Finalmente, el análisis de las repuestas con porcentajes de aparición superiores al $90 \%$, ha permitido apreciar ítems que no discriminan a los sujetos objeto de estudio, es decir, ítems propios de los sujetos analizados, y por lo tanto innecesarios (Zhu, Ennis, y Chen, 1998).

Con respecto a la fiabilidad, los datos del presente estudio indican la utilidad y necesidad de la prueba test-retest, en el proceso de elaboración de cuestionarios de esta índole en ciencias de la actividad física y el deporte, pruebas señaladas como necesarias por diferentes autores (Baumgartner, 2000; Carretero-Dios, y Pérez, 2007; Nevil, y col., 2001; Silverman y Subramanian, 1999).

Al profundizar en cada uno de los instrumentos, al observar el cuestionario de la percepción de la actitud del profesor/a en clase, los datos obtenidos en el presente estudio indican la necesidad de realizar modificaciones en ciertos ítems que no alcanzan las valoraciones exigidas (cuantitativa), en ciertos ítems que se aprecia dificultad en su comprensión, y en ciertos ítems que muestran porcentajes de respuesta elevados en comparación con el resto de ítems. Estos cambios (anexo 2) fueron realizados siguiendo las aportaciones de jueces expertos, las opiniones de los sujetos del estudio piloto, aspectos didácticos-deportivos (Silverman y Subramanian, 1999; Papaioannou y col., 2007), y aspectos metodológicos (Heinemann, 2003; Burgos, 2006). En cualquier caso, y a pesar de obtener valoraciones bajas por parte de los jueces expertos, se decidió mantener y modificar ligeramente los ítems 10 y 11 , debido a que la baja valoración, no se debía a la poca importancia del ítems, o a la falta de compresión, sino a la dificultad que conlleva la evaluación y valoración de las preguntas abiertas.

Por otro lado, al observar el cuestionario de la actitud del alumno en clase, los datos obtenidos indican la necesidad de mejorar ciertos aspectos, la adecuación al concepto fundamental sobre el que versa el planteamiento del instrumento «la actitud», la calidad y precisión respecto a algunos de los ítems, la adecuación específica del contenido a la estructura seguida. Los cambios fueron realizados siguiendo las aportaciones de jueces expertos, las opiniones de los sujetos del estudio piloto, aspectos didácticos-deportivos, $\mathrm{y}$ aspectos metodológicos. El principal, cambio se llevo a cabo modificando y adaptando a las sugerencias de los jueces expertos la tercera pregunta del segundo bloque, así como una reestructuración conceptual en la introducción del cuestionario.

\section{Coclusiones}

A partir de los resultados obtenidos se pueden establecer las siguientes conclusiones:

1) El cuestionario para evaluar la actitud del profesor en clase, dispone de niveles óptimos de validez y fiabilidad para registrar la percepción que tienen los alumnos sobre la actitud del profesor en clase (dominio sobre la materia que mostraba, evaluación que realizaba, y actitud del profesor en las relaciones sociales dentro de clase).

2) El cuestionario para evaluar la actitud del alumno en clase, dispone de niveles óptimos de validez y fiabilidad para registrar el grado de actitud de los alumnos de educación física de enseñanza secundaria (normas y reglamento, calentamiento previo, posibilidades física y capacidades de superación personal, ayuda y cooperación entre compañeros y profesor, efectos de la práctica sobre la salud, y actitud ante la clase de educación física).

\section{Referencias}

Ahrabi-Fard, I., \& Mavienko, O. A., (2005). Promoción de una educación activa de la actividad física orientada a la salud en las clases de educación física. Cultura, Ciencia y Deporte, 3(1), 163-170. Murcia: Universidad Católica San Antonio.

Altman, D. G. (1991). Practical statistics for medical research. New York: Chapman and Hall.

Baumgartner, T. A. (2000). Estimating the stability reliability of a store. Measurement in Physical Education and exercise Science, 4(3), $175-178$

B.O.E. (2000). REAL DECRETO 112/2000, de 28 de enero, por el que se crea la Comisión Nacional para la Protección de la Salud del Deportista.

B.O.E. (2006). REAL DECRETO 1631/2006, de 29 de diciembre, por el que se establecen las enseñanzas mínimas correspondientes a la Educación Secundaria Obligatoria.

B.O.R.M. (2002). Decreto 112/2002, de 13 de septiembre, por el que se establece el currículo de la Educación Secundaria Obligatoria en la Comunidad Autónoma de la Región de Murcia

Bulger, S.M., \& Housner, L.D. (2007). Modified delphi investigation of exercise science in physical education teacher education. Journal of Teaching in Physical Education, 26, 57-80

Bunner, G. (1997). Introduciendo a los niños en el atletismo a través de actividades de juego y aprendizaje. Cuadernos de Atletismo de la RFEA, 39, 41-49.

Bunner, G. (1999). Introducción de los niños en el atletismo a través del juego. Manual de atletismo en pabellones. Cuadernos de Atletismo de la RFEA, 46, 169-215.

Burgos, R. (2006). Metodología de investigación y escritura científica en clínica. Granada: Escuela Andaluza de Salud Pública.

Calderón, A., Palao, J. M., \& Ortega, E. (2005). Incidencia de la forma de organización sobre la participación, el feedback impartido, la calidad de las ejecuciones y la motivación en la enseñanza de habilidades atléticas. Cultura, Ciencia y Deporte, 3, 145-155. Murcia: Universidad Católica San Antonio.

Calvert, S., Ross, J., \& Hamlin, M. (2001). Levels of physical activity of a sample of 10-13 years old New Zealand children. New Zealand Medicine Journal. 114(1143), 496-498.

Carretero-Dios, H., \& Pérez, C. (2007). Normas para el desarrollo y revisión de estudios instrumentales: consideraciones sobre la selección de test en la investigación psicológica. International Journal of Clinical and Health Psychology, 7(3), 863-882.

Casimiro, A. J. (1999). Comparación, evolución y relación de hábitos saludables y nivel de condición fisica-salud en escolares entre final de educación primaria (12 años) y final de la educación secundaria obligatoria (16 años). [Tesis Doctoral]. Universidad de Granada.

Castejón, F. J., Giménez, F. J., Jiménez, F., \& López, V. (2003). Iniciación deportiva. La enseñanza y el aprendizaje comprensivo en el deporte. Sevilla: Wanceulen.

Cavill, N., Biddle, S. J. H., \& Sallis, J. F. (2001). Health enhancing physical activity for young people: Statement of the United Kingdom Expert Consensus Conference. Pediatric Exercise Science, 13, 12-25.

Ericsson, K. A. (1996). The road to excellence: the acquisition of expert performance in the arts and sciences sports and games. Erlbaum: Mahwah, New Jersey. 
Graham, G., Soares, P., \& Harrington, W. (1983). Experienced teachers effectiveness in children's physical education. Journal of Teaching in Physical Education, 2, 3-14

Hassandra, M., Goudas, M., \& Chroni, S. (2003). Examining factors associated with intrinsic motivation in physical education: a qualitative approach. Psychology of Sport and Exercise, 4, 211-223.

Heinemann, K. (2003). Introducción a la metodología de la investigación empírica en las ciencias del deporte. Barcelona: Paidotribo

Hernández, J.M., Velázquez, R., Alonso, D., Castejón, F.J., Garoz, I., López, C., López, Angeles, Maldonado, A., y Martínez, M.E. (2004). La evaluación en educación física : investigación y práctica en el ámbito escolar. Grao Editorial: Barcelona

Herrero, M. L. (2002). Introducción a los métodos, diseños y técnicas de investigación psicológicas. Zaragoza: textos docentes

Magalhães, M., \& Hill, A. (2005). Investigação por questionário. Lisboa: Sílabo.

Nevil, A.M., Lane, A.M., Kilgour, L.J., Bowes, N., \& Whyte, G.P. (2001) Stability of psychometric questionnaires. Journal of Sports Science, 19, 273-278

Ntoumanis, N., Pensgaard, A. M., Martin, C., \& Pipe, K. (2004). An Idiographic Analysis of Amotivation in Compulsory School Physical Education. Journal of Sport \& Exercise Psychology, 26, 197-214.

Papaioannou, A.G., Tsigilis, N. Kosmidou, E. \& Milosis, D. (2007). Measuring Perceived Motivational Climate in Physical Education. Journal of Teaching in Physical Education, 26, 236-259

Pierón, M. (1988). Didáctica de las actividades fisico deportivas. Gymnos: Madrid.

Ramos-Álvarez, M.M., Valdés-Conroy, B. y Catena, A. (2006). Criteria of the peer-review process for publication of experimental and cuasi- experimental research in Psychology. International Journal of Clinical and Health Psychology, 6, 773-787.

Sallis, J. F., Mckenzie, T. L., \& Alcaráz, F. E. (1993). Habitual Physical activity and health-related physical fitness in fourth-grade children. American Journal of deseases in childhood, 147, 890-896.

Silverman, S. (1985). Relationship of engagement and practice trials to student achievement. Journal of Teaching in Physical Education, 5, 13-21.

Silverman, S., \& Subramanian, P.R. (1999). Student attitude toward education and physical activity: a review of measurement issues and outcomes. Journal of Teaching in Physical Education, 19, 97-125.

Swain, D. P., \& Franklin, B. A. (2002). VO(2) reserve and the minimal intensity for improving cardiorespiratory fitness. Medicine Science Sports Exercise. 34(1), 152-157.

Thomas, J. R., \& Nelson, J. K. (2007). Métodos de investigación en actividad fisica. Barcelona: Paidotribo.

Ureña, F., Soriano, L. M., Martínez, A., \& Hernández, M. A. (1997). La Educación Física en Secundaria. Fundamentación teórica. Colección educación física en la reforma. Barcelona: Inde.

US Departament of health and human services (2000). Healthy people 2010. Washington, DC. http:/healthypeople.gov/document/, accedido 16 julio, 2007.

\section{VERSIÓN FINAL DEL CUESTIONARIO DE ACTITUD PERCIBIDA POR LOS ALUMNOS DEL} PROFESOR EN CLASE DE EDUCACIÓN FÍSICA (Anexo 2)

Este cuestionario está pensado para ayudar a tu profesor a mejorar la forma de dar las clases. Para contestar sólo tienes que seguir la escala que te proponemos:

\begin{tabular}{|c|c|c|c|}
\hline $1=$ No adecuado & $2=$ Regular & $3=$ Bueno & $4=$ Muy bueno \\
\hline
\end{tabular}

Rodea con un círculo el valor que consideres más apropiado en relación con tu percepción de la actuación de tu profesor o profesora. Si te equivocas puedes tacharlo y rodear otro número.

\begin{tabular}{|l|l|c|c|c|c|}
\hline & & $\begin{array}{c}\text { No } \\
\text { adecuado }\end{array}$ & Regular & Bueno & $\begin{array}{c}\text { Muy } \\
\text { Bueno }\end{array}$ \\
\hline $\mathbf{1}$ & Explicando la materia en clase teórica lo consideras: & $\mathbf{1}$ & $\mathbf{2}$ & $\mathbf{3}$ & $\mathbf{4}$ \\
\hline $\mathbf{2}$ & Explicando la materia en clase práctica lo consideras: & $\mathbf{1}$ & $\mathbf{2}$ & $\mathbf{3}$ & $\mathbf{4}$ \\
\hline $\mathbf{3}$ & Realizando una demostración de la destreza lo consideras & $\mathbf{1}$ & $\mathbf{2}$ & $\mathbf{3}$ & $\mathbf{4}$ \\
\hline $\mathbf{4}$ & El control y organización de la clase es: & $\mathbf{1}$ & $\mathbf{2}$ & $\mathbf{3}$ & $\mathbf{4}$ \\
\hline $\mathbf{5}$ & El procedimiento de evaluación es: & $\mathbf{1}$ & $\mathbf{2}$ & $\mathbf{3}$ & $\mathbf{4}$ \\
\hline $\mathbf{6}$ & La forma en la que se relaciona con los alumnos es: & $\mathbf{1}$ & $\mathbf{2}$ & $\mathbf{3}$ & $\mathbf{4}$ \\
\hline $\mathbf{7}$ & La atención a los problemas individuales es: & $\mathbf{1}$ & $\mathbf{2}$ & $\mathbf{3}$ & $\mathbf{4}$ \\
\hline $\mathbf{8}$ & En sus clases el clima de convivencia es & $\mathbf{1}$ & $\mathbf{2}$ & $\mathbf{3}$ & $\mathbf{4}$ \\
\hline $\mathbf{9}$ & Lo que más te gusta de la forma de llevar las clases por parte del profesor es: & & \\
\hline $\mathbf{1 0}$ & Lo que menos te gusta de la forma de llevar las clases por parte del profesor es: & \\
\hline $\mathbf{1 1}$ & Que aconsejarías al profesor para mejorar sus clases: & \\
\hline
\end{tabular}

Weinberg, R. S., \& Gould, D. (1995). Foundations of sport and exercise psychology. Champaign: Human Kinetics.

Wiersma, L.D. (2001). Conceptualization and development of the sources of enjoyment in youth sport questionnaire. Measurement in Physical Education and Exercise Science, 5(3), 153-177.

Xiang, P., McBride, R., \& Guan, J. (2004). Children's motivation in elementary physical education: A longitudinal study. Research Quarterly for Exercise and Sport, 75(1), 71-80.

Zhu, W., Ennis, C. D., \& Chen, A. (1998). Many-faceted rasch modelling expert judgment in test development. Measurement in Physical Education and Exercise Science, 2(1), 21-39 


\section{VERSIÓN INICIAL DEL CUESTIONARIO PARA VALORAR LA ACTITUD DEL ALUMNO EN CLASE DE EDUCACIÓN FÍSICA (Anexo 3)}

El presente cuestionario pretende conocer diferentes elementos que confirman el proceso de enseñanza-aprendizaje. Por favor sé sincero en tus respuestas.

\begin{tabular}{|c|c|c|c|c|}
\hline 1. En relación con las normas y el reglamento: & Siempre & A menudo & A veces & Nunca \\
\hline $\begin{array}{l}\text {-Es preciso seguir el reglamento y las normas de juego (aunque esto no me } \\
\text { permita ganar siempre). }\end{array}$ & A & B & $\mathrm{C}$ & $\mathrm{D}$ \\
\hline $\begin{array}{l}\text {-Ganar en un equipo o juego, solo tiene mérito cuando se respetan las reglas del } \\
\text { juego. }\end{array}$ & A & B & $\mathrm{C}$ & $\mathrm{D}$ \\
\hline -Los árbitros siempre tratan de emitir sus juicios sin favorecer a nadie. & A & B & $\mathrm{C}$ & $\mathrm{D}$ \\
\hline 2. En relación con el calentamiento previo a la práctica de ejercicio fisico: & Siempre & A menudo & A veces & Nunca \\
\hline $\begin{array}{l}\text {-Es necesario realizar un buen calentamiento, porque así preparo mi cuerpo para } \\
\text { afrontar la actividad posterior. }\end{array}$ & A & B & $\mathrm{C}$ & $\mathrm{D}$ \\
\hline -Normalmente, sólo hago ejercicios de calentamiento cuando hace frío. & A & $\mathrm{B}$ & $\mathrm{C}$ & $\mathrm{D}$ \\
\hline $\begin{array}{l}\text {-Sólo realizo el calentamiento en clase de educación física con el profesor, } \\
\text { cuando hago ejercicios por mi cuenta, considero que no hace falta. }\end{array}$ & A & B & $\mathrm{C}$ & D \\
\hline 3. En relación con tus posibilidades físicas y tu capacidad de auto-superación: & Siempre & A menudo & A veces & Nunca \\
\hline $\begin{array}{l}\text {-Siempre trato de esforzarme para tratar de aprender habilidades y gestos nuevos } \\
\text { en clase. }\end{array}$ & A & B & $\mathrm{C}$ & $\mathrm{D}$ \\
\hline $\begin{array}{l}\text {-En muchas ocasiones me desanimo porque no me sale lo que el profesor plantea } \\
\text { en clase de educación física. }\end{array}$ & A & B & $\mathrm{C}$ & D \\
\hline $\begin{array}{l}\text {-En las clases de educación física puedo resolver las tareas difíciles si me } \\
\text { esfuerzo lo suficiente. }\end{array}$ & A & & $\mathrm{C}$ & D \\
\hline $\begin{array}{l}\text {-Gracias a mis cualidades y recursos puedo superar situaciones imprevistas en la } \\
\text { práctica de actividades físicas. }\end{array}$ & A & & $\mathrm{C}$ & $\mathrm{D}$ \\
\hline $\begin{array}{l}\text {-Pase lo que pase durante la práctica deportiva por lo general soy capaz de } \\
\text { controlar la situación. }\end{array}$ & 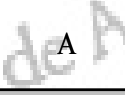 & 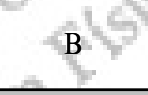 & $\mathrm{C}$ & D \\
\hline $\begin{array}{l}\text { 4. En relación con las ayudas y la cooperación entre compañeros y con el } \\
\text { profesor: }\end{array}$ & Siempre & A menudo & A veces & Nunca \\
\hline $\begin{array}{l}\text {-Ayudo a mis compañeros siempre que necesiten ayuda mediante consejos, } \\
\text { ánimo, correcciones, ayudas manuales, etc. }\end{array}$ & A & B & $\mathrm{C}$ & $\mathrm{D}$ \\
\hline $\begin{array}{l}\text {-Ayudo al profesor a sacar y guardar el material, ya que eso también es labor } \\
\text { nuestra. }\end{array}$ & A & B & $\mathrm{C}$ & $\mathrm{D}$ \\
\hline $\begin{array}{l}\text { - Pase lo que pase durante la práctica deportiva soy capaz de mantener valores } \\
\text { de compañerismo. }\end{array}$ & A & B & $\mathrm{C}$ & D \\
\hline $\begin{array}{l}\text { 5. En relación con los efectos que la práctica habitual de actividad fisica } \\
\text { produce sobre la salud: }\end{array}$ & $\begin{array}{l}\text { Nada de } \\
\text { acuerdo }\end{array}$ & $\begin{array}{l}\text { Algo de } \\
\text { acuerdo }\end{array}$ & $\begin{array}{l}\text { Bastante de } \\
\text { acuerdo }\end{array}$ & $\begin{array}{l}\text { Totalmente de } \\
\text { acuerdo }\end{array}$ \\
\hline -Hago deporte y lo practico siempre que puedo ya que favorecerá mi salud. & A & B & $\mathrm{C}$ & $\mathrm{D}$ \\
\hline $\begin{array}{l}\text {-La actividad física y deportiva solo la practico por obligación, no creo que } \\
\text { favorezca mi salud. }\end{array}$ & A & B & $\mathrm{C}$ & $\mathrm{D}$ \\
\hline -Me encanta la actividad deportiva pero solo para verla como espectador. & A & B & $\mathrm{C}$ & $\mathrm{D}$ \\
\hline -Me encanta la actividad deportiva pero no puedo practicarla por falta de tiempo. & A & B & $\mathrm{C}$ & $\mathrm{D}$ \\
\hline 6. Cuando voy a clase de educación física... & Nunca & Pocas veces & $\begin{array}{c}\text { Bastantes } \\
\text { veces }\end{array}$ & Siempre \\
\hline -Voy pensando que voy a mejorar mi estado de ánimo. & A & B & $\mathrm{C}$ & $\mathrm{D}$ \\
\hline -Voy pensando que el profesor-a suele hacerme poco caso. & A & B & $\mathrm{C}$ & $\mathrm{D}$ \\
\hline -Voy pensando que es un buen momento de relacionarme con los demás. & A & B & $\mathrm{C}$ & $\mathrm{D}$ \\
\hline $\begin{array}{l}\text {-Voy pensando que el profesor-a suele animarme para participar en las } \\
\text { actividades. }\end{array}$ & A & B & $\mathrm{C}$ & $\mathrm{D}$ \\
\hline -Voy pensando que es importante para mi desarrollo como persona. & A & B & $\mathrm{C}$ & $\mathrm{D}$ \\
\hline Otra ¿cuál?. & A & B & $\mathrm{C}$ & $\mathrm{D}$ \\
\hline
\end{tabular}




\section{ESCALA DE LA ACTITUD DEL ALUMNO EN CLASE (Anexo 4)}

El presente cuestionario pretende valorar la actitud del alumno hacia la práctica de actividad física en general, y hacia las clases de educación física en particular. Rodea con una única opción cada pregunta. Por favor sé sincero en tus respuestas.

\begin{tabular}{|c|c|c|c|c|}
\hline 1.- En relación con las normas y reglas: & Siempre & A menudo & A veces & Nunca \\
\hline $\begin{array}{l}\text {-Es preciso seguir el reglamento y las normas (aunque esto no me permita } \\
\text { ganar siempre o lograr los objetivos). }\end{array}$ & A & B & $\mathrm{C}$ & $\mathrm{D}$ \\
\hline $\begin{array}{l}\text {-Ganar o cumplir los objetivos, solo tiene mérito cuando se respetan las } \\
\text { reglas y las normas. }\end{array}$ & A & B & $\mathrm{C}$ & $\mathrm{D}$ \\
\hline $\begin{array}{l}\text {-Los árbitros o jueces siempre tratan de emitir sus juicios sin favorecer a } \\
\text { nadie. }\end{array}$ & A & B & $\mathrm{C}$ & $\mathrm{D}$ \\
\hline $\begin{array}{l}\text { 2.- En relación con el calentamiento previo a la práctica de ejercicio } \\
\text { fisico: }\end{array}$ & Siempre & A menudo & A veces & Nunca \\
\hline $\begin{array}{l}\text {-Hago un buen calentamiento, porque así preparo mi cuerpo para afrontar } \\
\text { la actividad posterior }\end{array}$ & A & B & $\mathrm{C}$ & $\mathrm{D}$ \\
\hline -Normalmente, sólo hago ejercicios de calentamiento cuando hace frío. & A & B & $\mathrm{C}$ & $\mathrm{D}$ \\
\hline $\begin{array}{l}\text { - No lo considero necesario, por lo que cuando practico actividad física por } \\
\text { mi propia cuenta no lo realizo; sólo lo pongo en práctica cuando el profesor } \\
\text { lo propone en las clases de educación física }\end{array}$ & & B & $\mathrm{C}$ & $\mathrm{D}$ \\
\hline $\begin{array}{l}\text { 3.- En relación con tus posibilidades fisicas y tu capacidad de auto- } \\
\text { superación: }\end{array}$ & Siempre & A menudo & A veces & Nunca \\
\hline $\begin{array}{l}\text {-Siempre me esfuerzo para tratar de aprender habilidades y gestos nuevos } \\
\text { en clase. }\end{array}$ & A & B & $\mathrm{C}$ & $\mathrm{D}$ \\
\hline $\begin{array}{l}\text {-Me desanimo frecuentemente porque no me sale lo que el profesor plantea } \\
\text { en clase de educación física. }\end{array}$ & A & & $\mathrm{C}$ & $\mathrm{D}$ \\
\hline $\begin{array}{l}\text {-Soy capaz de resolver las tareas difíciles si me esfuerzo lo suficiente y lo } \\
\text { sé. }\end{array}$ & A & & 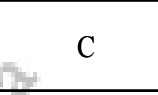 & $\mathrm{D}$ \\
\hline $\begin{array}{l}\text {-Gracias a mis cualidades y recursos puedo superar situaciones imprevistas } \\
\text { en la práctica de actividades físicas. }\end{array}$ & & & $\mathrm{C}$ & $\mathrm{D}$ \\
\hline $\begin{array}{l}\text { - Durante la práctica deportiva por lo general soy capaz de controlar la } \\
\text { situación pase lo que pase. }\end{array}$ & Lit & & $\mathrm{C}$ & $\mathrm{D}$ \\
\hline $\begin{array}{l}\text { 4.- En relación con las ayudas y la cooperación entre compañeros y con } \\
\text { el profesor: }\end{array}$ & Siempre & A menudo & A veces & Nunca \\
\hline $\begin{array}{l}\text {-Ayudo a mis compañeros siempre que necesiten ayuda mediante consejos, } \\
\text { ánimo, correcciones, ayudas manuales, etc. }\end{array}$ & A & B & $\mathrm{C}$ & $\mathrm{D}$ \\
\hline $\begin{array}{l}\text {-Ayudo al profesor a sacar y guardar el material, ya que eso también es } \\
\text { labor nuestra. }\end{array}$ & A & B & $\mathrm{C}$ & $\mathrm{D}$ \\
\hline $\begin{array}{l}\text { - Durante la práctica deportiva soy capaz de mantener valores de } \\
\text { compañerismo pase lo que pase. }\end{array}$ & A & B & $\mathrm{C}$ & $\mathrm{D}$ \\
\hline $\begin{array}{l}\text { 5.- En relación con los efectos que la práctica habitual de actividad fisica } \\
\text { produce sobre la salud: }\end{array}$ & $\begin{array}{l}\text { Nada de } \\
\text { acuerdo }\end{array}$ & $\begin{array}{l}\text { Algo de } \\
\text { acuerdo }\end{array}$ & $\begin{array}{c}\text { Bastante de } \\
\text { acuerdo }\end{array}$ & $\begin{array}{l}\text { Totalmente de } \\
\text { acuerdo }\end{array}$ \\
\hline -Hago deporte y lo practico siempre que puedo ya que favorecerá mi salud. & A & B & $\mathrm{C}$ & $\mathrm{D}$ \\
\hline $\begin{array}{l}\text {-La actividad física y deportiva solo la practico por obligación, no creo que } \\
\text { favorezca mi salud. }\end{array}$ & A & B & $\mathrm{C}$ & $\mathrm{D}$ \\
\hline $\begin{array}{l}\text {-La práctica de actividad física y deportiva regular solo es beneficiosa si se } \\
\text { práctica de forma regular al menos tres veces por semana durante una hora. }\end{array}$ & A & B & $\mathrm{C}$ & $\mathrm{D}$ \\
\hline 6.- Cuando voy a clase de educación física... & Nunca & Pocas veces & $\begin{array}{c}\text { Bastantes } \\
\text { veces }\end{array}$ & Siempre \\
\hline -Voy pensando que voy a mejorar mi estado de ánimo. & A & B & $\mathrm{C}$ & $\mathrm{D}$ \\
\hline -Voy pensando que el profesor/a suele hacerme poco caso. & A & $\mathrm{B}$ & $\mathrm{C}$ & $\mathrm{D}$ \\
\hline -Voy pensando que es un buen momento de relacionarme con los demás. & A & B & $\mathrm{C}$ & $\mathrm{D}$ \\
\hline $\begin{array}{l}\text {-Voy pensando que el profesor-a suele animarme para participar en las } \\
\text { actividades. }\end{array}$ & A & B & $\mathrm{C}$ & $\mathrm{D}$ \\
\hline -Voy pensando que es importante para mi desarrollo como persona. & A & $\mathrm{B}$ & $\mathrm{C}$ & $\mathrm{D}$ \\
\hline - Otra ¿cuál?. & $\mathrm{A}$ & B & $\mathrm{C}$ & $\mathrm{D}$ \\
\hline
\end{tabular}

